\title{
Infrared Laser Excitation to Study Transitions in Fullerene (C60) Molecule
}

\section{Subramaniam TY* and Premanand $\mathbf{P}$}

Department of Science and Humanities (Physics), Sri Sairam Engineering College, Chennai, India

\begin{abstract}
Raman spectroscopy is the study of inelastic scattering of light. A Raman active sample should show a change in polarizability. It provides amongst other things, important information about the vibrational state of matter. Raman studies in scattering entails a sum of both creation and annihilation of photons and law of energy conservation applies to only the overall process and not to individual events. Infrared laser beam is a good choice for studying absorption and emission characteristics of fullerene molecule. The photo physical, photochemical and optical properties have already been studied in detail by many scientists. We intend to study the infrared laser induced characteristics of fullerene molecule. It is expected that laser interaction with $\mathrm{C}_{60}$ molecule can break the carbon bond, creating more free electrons resulting in formation of new compounds.
\end{abstract}

Keywords: Inelastic scattering; Vibrational state; Photo physical; Photochemical; Fullerene molecule; Infrared laser

\section{Introduction}

The fullerene molecule $\mathrm{C}_{60}$ was discovered by Kroto, et al. [1] $\mathrm{C}_{60}$ has much weaker inter-molecular forces than diamond, another allotrope of carbon. It has a much lower melting point and boiling point than diamond. Therefore, less energy is required to break the forces of attraction. Raman spectroscopy was named in the honour of its discoverer Sir. Raman CV, who along with Krishnan KS published the first paper on this technique. Raman spectroscopy (RS) is a versatile method for analysis of a wide range of samples. It resolves most of the limitations of other spectroscopic techniques. Qualitative analysis can be performed by measuring the frequency of scattered radiation while quantitative analysis can be performed by measuring the intensity of the scattered radiation. Raman spectroscopy is basically a light scattering technique. The frequency of a small fraction of the scattered radiation is found to be different from the frequency of a monochromatic incident radiation [2]. It probes molecular vibrations. After the excitation, for the total energy of the system to remain constant even after the molecule moves to a new rovibronic state, the scattered photon shifts to a different energy, and therefore a different frequency can be seen [3] (Figure 1).

\section{Experiment}

In our Raman spectroscopy experiment, the sample $\mathrm{C}_{60}$ is illuminated with a monochromatic laser, (Nd: YAG laser beam with a wavelength of $\lambda=1.064 \mu \mathrm{m}$ ) which interacts with the $C_{60}$ molecules and generated a scattered radiation. Thus, the scattered light, having a wavelength different from that of the incident wavelength is used to construct a Raman spectrum. This type of scattering (inelastic) arises due to the inelastic collisions between the incident monochromatic radiation and the molecules of the $\mathrm{C}_{60}$. We has given the sample containing $\mathrm{C}_{60}$, pure (99.99\%), research grade, nano particles obtained from M/s. Merck and Co. (formerly Sigma-Aldrich) for analysis with an instrument named Bruker RFS27, at the Sophisticated Analysis Instrumentation Facility (SAIF), Indian Institute of Technology (IIT-M), Chennai. The sample analysis was performed from the wave number range of $4000 \mathrm{~cm}^{-1}$ to $400 \mathrm{~cm}^{-1}$. Raman spectrophotometers can be either a dispersive or a non-dispersive unit. In a dispersive unit, a prism or a grating will be used for analysis. In a non-dispersive unit, a Michelson interferometer is used. In FT-Raman, an interferometer is used for analysis.

\section{Analysis}

The spectrum recorded is shown here below, in Figure 2 below. In the above spectrum, the peaks seen at $1467.30 \mathrm{~cm}^{-1}, 493.96 \mathrm{~cm}^{-1}, 269.36$ $\mathrm{cm}^{-1}$, are due to resonance Raman lines ( $\mathrm{Q}$ branch), which goes to highest values recorded in any spectrum. These could not be attributed to Rayleigh scattering, since the recorded spectrum itself is about Raman intensity only and not recorded in any arbitrary unit (a.u).The peaks recorded at $1573.59 \mathrm{~cm}^{-1}, 2841.02 \mathrm{~cm}^{-1}, 1420.51 \mathrm{~cm}^{-1}, 1264.94 \mathrm{~cm}$ ${ }^{1}, 1099.23 \mathrm{~cm}^{-1}, 771.25 \mathrm{~cm}^{-1}, 566.95 \mathrm{~cm}^{-1}$, and at $431.15 \mathrm{~cm}^{-1}$ are all due to either Stokes shift or due to anti-Stokes shift lines. The resonance Raman lines (RR) is a name given to a laser frequency which is close in energy to an electronic transition of a compound or a crystal under examination. This frequency can lead to a greatly enhanced intensity of the Raman scattering, which facilitates the study of compounds present at low concentrations [4]. In a Raman spectrum, Q branch will be present and the same will be absent in an infrared spectrum. The $\mathrm{O}$ and the $\mathrm{S}$ branches are similar to the $\mathrm{R}$ and $\mathrm{P}$ branches in an infrared spectrum, as shown in Figure 3 below.

The selection rules for the Stokes and anti-Stokes shift are as follows:

$\Delta \mathrm{J}=+2$ and $\Delta v=+1$ gives $\mathrm{S}$ branch in a Raman spectrum, corresponding to Stokes shift;

$\Delta \mathrm{J}=0$, and $\Delta v=+1$ gives the $\mathrm{Q}$ branch, corresponding to the Resonance Raman (RR) lines in a Raman spectrum;

$\Delta \mathrm{J}=-2$ and $\Delta v=+1$ gives the $\mathrm{O}$ branch, corresponding to the antiStokes shift in a Raman spectrum.

Hence the Stokes lines and the anti-Stokes lines will be on either side of the resonance Raman lines in a Raman spectrum. Professor Gerhard

*Corresponding author: Subramaniam TY, Department of Science and Humanities (Physics), Sri Sairam Engineering College, Sairam Campus, Sai Leo Nagar, West Tambaram, Chennai, Tamil Nadu, India, 600044, Tel: 044-2251 2222; E-mail: subramaniam.phy@sairam.edu.in

Received May 30, 2018; Accepted June 27, 2018; Published July 04, 2018

Citation: Subramaniam TY, Premanand P (2018) Infrared Laser Excitation to Study Transitions in Fullerene (C60) Molecule. J Laser Opt Photonics 5: 189. doi: 10.4172/2469-410X.1000189

Copyright: (c) 2018 Subramaniam TY, et al. This is an open-access article distributed under the terms of the Creative Commons Attribution License, which permits unrestricted use, distribution, and reproduction in any medium, provided the original author and source are credited. 


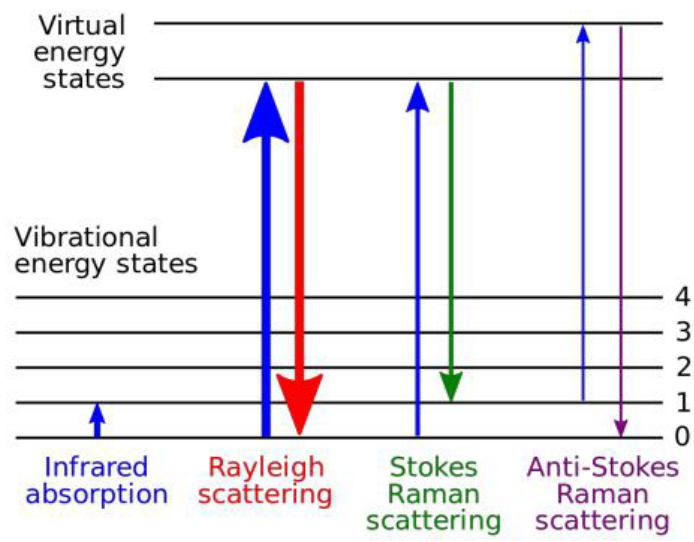

Figure 1: Stokes and anti-Stokes shift in Raman scattering.
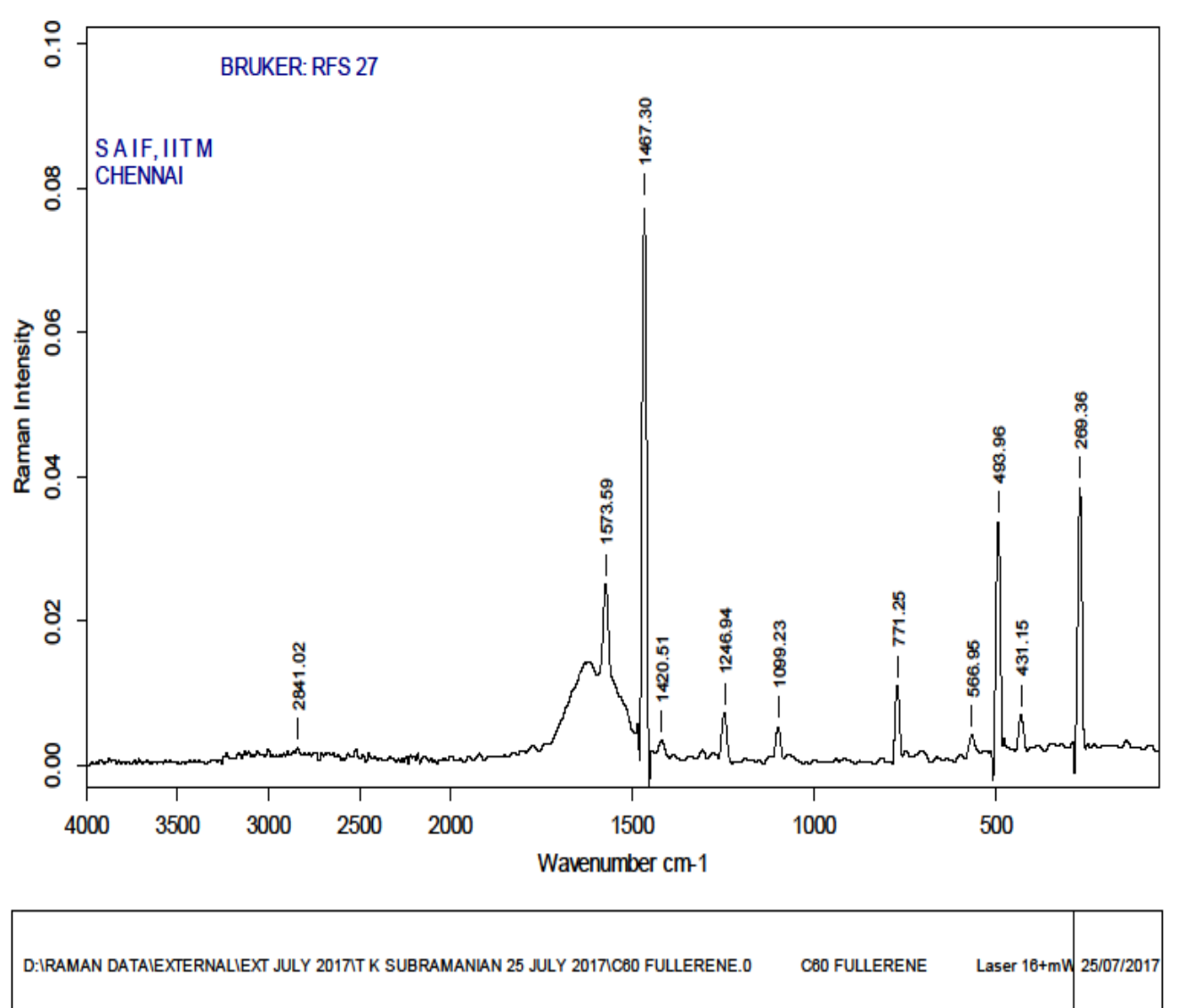

Page 1/1

Figure 2: FT-Raman spectrum recorded for C60 molecule between $4000 \mathrm{~cm}-1$ to $400 \mathrm{~cm}-1$.

Herzberg has contributed enormously to the study of molecules using different types of radiations present in the electromagnetic spectrum. He is regarded as the Father of Molecular Spectroscopy. The anti-Stokes line is much less intense than the Stokes line. This occurs because only molecules that are vibrationally excited prior to irradiation can give rise to the anti-Stokes line. Hence, in Raman spectroscopy, only the more intense Stokes line is normally measured. Hence, from the recorded spectrum above, we find that the lines having the energies at 1573.59 $\mathrm{cm}^{-1}, 771.25 \mathrm{~cm}^{-1}, 431.15 \mathrm{~cm}^{-1}$ are attributed to the Stokes shift and the other lines, namely, $2841.02 \mathrm{~cm}^{-1}, 1420.51 \mathrm{~cm}^{-1}, 1246.94 \mathrm{~cm}^{-1}, 1099.23$ $\mathrm{cm}^{-1}, 566.95 \mathrm{~cm}^{-1}$ are attributed to the anti-Stokes shift.

It is not necessary that all the Raman lines will have their corresponding infrared bands and similarly, all infrared bands may not have their corresponding Raman lines. Generally, Raman lines are symmetrically displaced about the parent lines. When the temperature increases, their individual separations from the parent lines decrease. In order to find more anti-Stokes lines that are present in the spectrum 


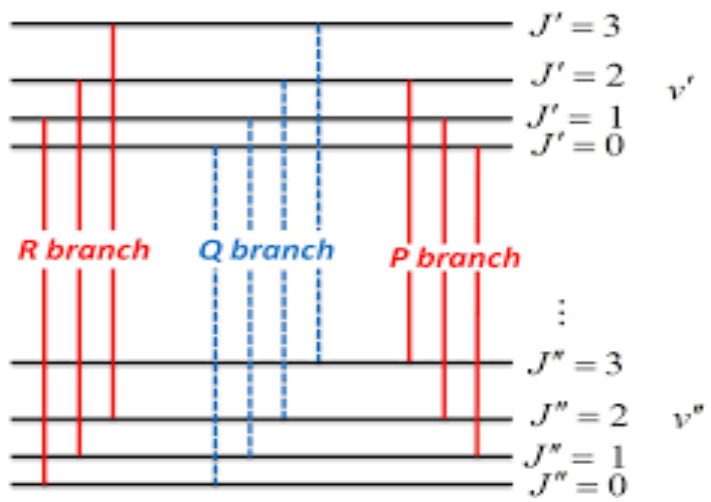

Figure 3: $\mathrm{P}$ and $\mathrm{R}$ branches in an infrared spectrum and $\mathrm{Q}$ branch (in dotted lines) in a Raman spectrum for a molecule.

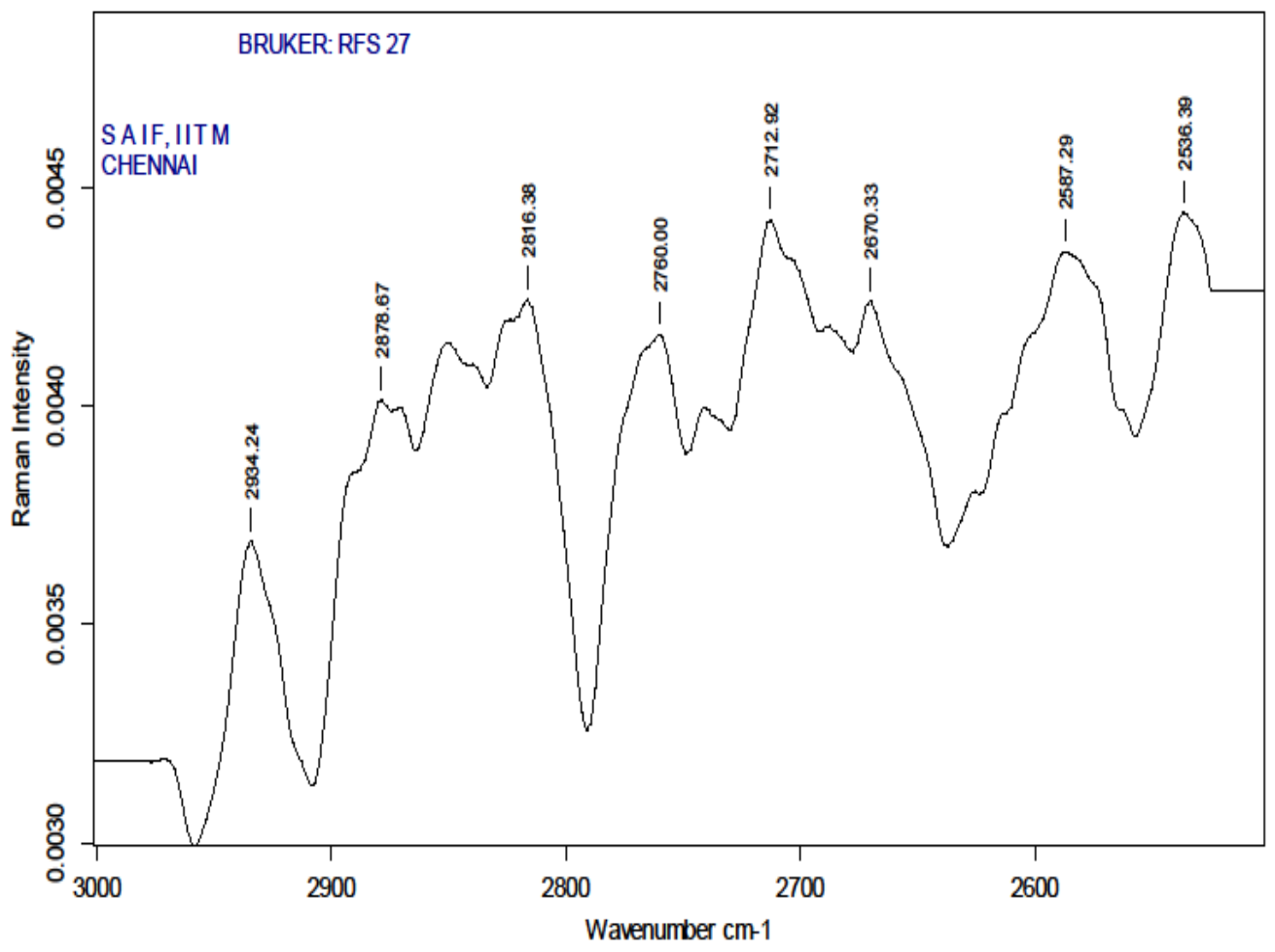

D:IRAMAN DATAIEXTERNALIEXT JULY 2017TT K SUBRAMANIAN 25 JULY 2017/C80 FULLERENE REP 1.0 C60 FULLERENE REP 1

$25 / 07 / 2017$

Page 1/1

Figure 4: Raman spectrum of C60 molecule recorded between $3000 \mathrm{~cm}-1$ to $2500 \mathrm{~cm}-1$ as an expanded plot.

that we have recorded, we took an expanded plot between $3000 \mathrm{~cm}^{-1}$ to $2500 \mathrm{~cm}^{-1}$ region. The recorded spectrum is shown here below (Figure 4).

Whereas only one line could be seen between $3000 \mathrm{~cm}^{-1}$ to 2500 $\mathrm{cm}^{-1}$ in the spectrum as shown in Figure 4 above, we could see that from the Figure 5 above, there is more anti-Stokes lines present due to rovibronic transitions of the $\mathrm{C}_{60}$ molecule.

The intensities shown in the $\mathrm{Y}$-axis is evident that these lines could not surface in the earlier spectrum (Figure 3), because they were weak in intensity. This leads us to conclude that, on a laser excitation, the $\mathrm{C}_{60}$ molecule forms many smaller compounds from $\mathrm{C}_{60}$ itself, with a lower concentration.

The lines which are attributed to anti-Stokes shift are: $2934 \mathrm{~cm}$ ${ }^{1}, 2878.67 \mathrm{~cm}^{-1}, 2816.38 \mathrm{~cm}^{-1}, 2760.00 \mathrm{~cm}^{-1}, 2712.92 \mathrm{~cm}^{-1}, 2670.33 \mathrm{~cm}^{-1}$, $2587.29 \mathrm{~cm}^{-1}$ and $2536.39 \mathrm{~cm}^{-1}$, respectively.

The rovibronic transitions are mainly due to the different stretching and bending modes that are present due to laser excitation of the $\mathrm{C}_{60}$ 


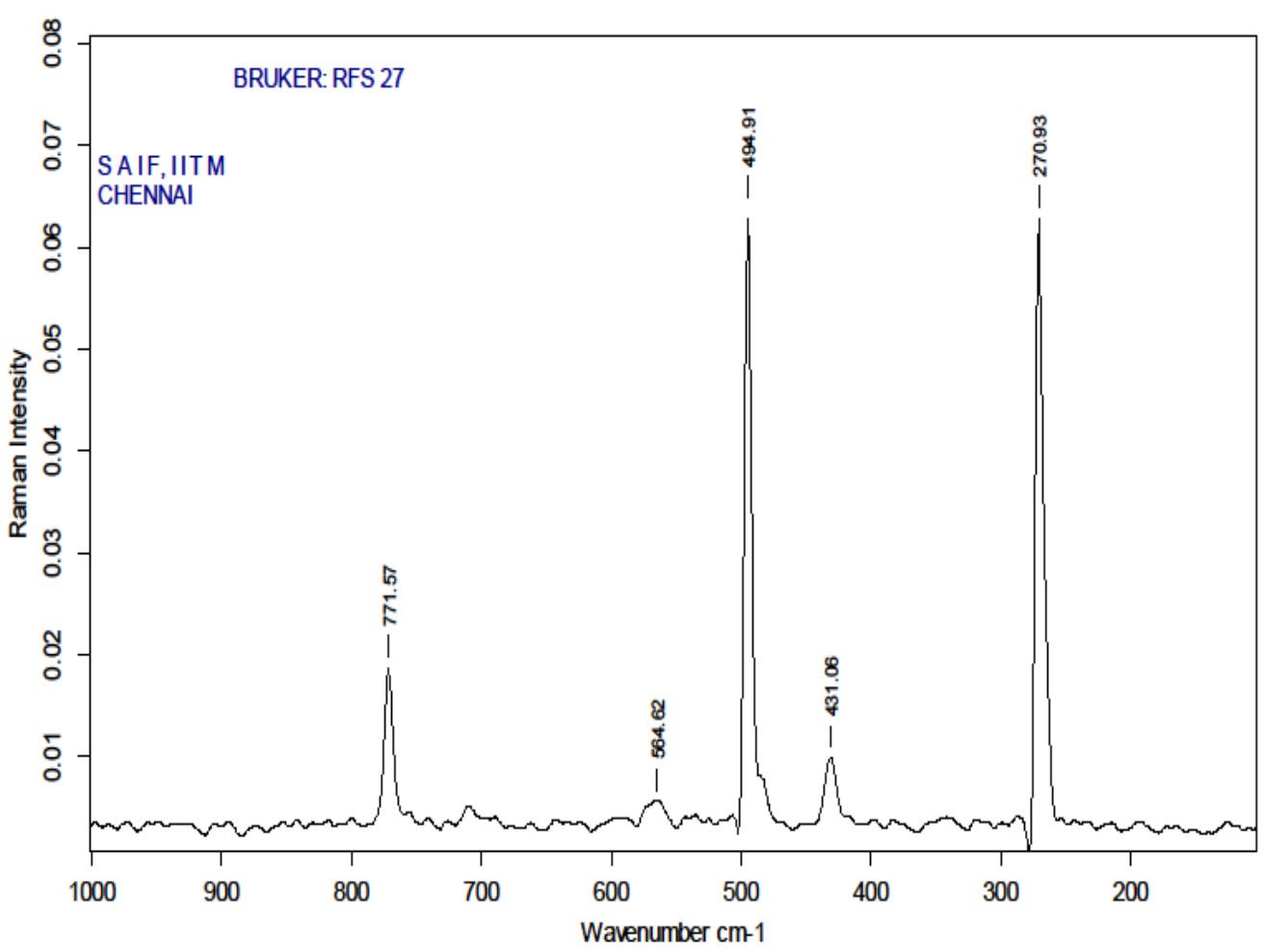

D:IRAMAN DATAIEXTERNALIEXT JULY 2017IT K SUBRAMANIAN 25 JULY 2017IC80 FULLERENE REP.0 I C CO FULLERENE REP

Page 1/1

Figure 5: FT-Raman lines between $1000 \mathrm{~cm}-1$ and $100 \mathrm{~cm}-1$ (finger print region).

molecule. The number of normal modes of vibration is $3 \mathrm{~N}-6$, where $\mathrm{N}$ is the number of atoms in a molecule.

Therefore, the total number of modes present is $3 \times 60-6=180$ $6=174$ modes. The normal modes are due to symmetric stretching, asymmetric stretching and bending modes. The equation for an harmonic oscillator is used to describe the energy of various levels in a molecule. The levels are unequally placed with the spacing between the energy levels decreasing, as it goes towards higher energies.

The equation governing the an harmonic oscillator is given by, $\mathrm{G}$ $(v)=\omega_{e}(v+1 / 2)-x_{e}$

$$
(v+1 / 2)^{2}+y_{e}(v+1 / 2)^{3}+\ldots
$$

Here, $x_{e}$ and $y_{e}$ are the an harmonic constants. The negative sign is placed in front of the second term for convenience so that all values of $\mathrm{x}_{\mathrm{e}}$ and $\mathrm{y}_{\mathrm{e}}$ are positive [5].

From the Figure 3, the lines due to $2841.02 \mathrm{~cm}^{-1}, 1573.59 \mathrm{~cm}^{-1}$, $1467.30 \mathrm{~cm}^{-1}$ are attributed to the $\mathrm{C}-\mathrm{H}, \mathrm{C}=\mathrm{O}, \mathrm{C}=\mathrm{N}, \mathrm{C}=\mathrm{C}$ stretching bonds.

From the Figure 5, we note that, the lines due to $2934 \mathrm{~cm}^{-1}, 2878.67$ $\mathrm{cm}^{-1}, 2816.38 \mathrm{~cm}^{-1}$, are due to $\mathrm{C}-\mathrm{H}$ stretching bond.

We also recorded a Raman spectrum in the famous "finger print" region $\left(1500 \mathrm{~cm}^{-1}\right.$ to $\left.100 \mathrm{~cm}^{-1}\right)$, to see the presence of Stokes and antiStokes shifts. The spectrum recorded is in the region between $1000 \mathrm{~cm}^{-1}$ to $100 \mathrm{~cm}^{-1}$, shown below (Figure 5).
The lines due to the energies, $1420.51 \mathrm{~cm}^{-1}$ (Stokes), $1246.94 \mathrm{~cm}^{-1}$ (anti-Stokes), $1099.23 \mathrm{~cm}^{-1}$ (anti-Stokes), $771.25 \mathrm{~cm}^{-1}$ (Stokes), 566.95 $\mathrm{cm}^{-1}$ (anti-Stokes), $493.96 \mathrm{~cm}^{-1}$ (Resonance Raman), $431.15 \mathrm{~cm}^{-1}$ (Stokes) and $269.36 \mathrm{~cm}^{-1}$ or $270.93 \mathrm{~cm}^{-1}$ (Resonance Raman) are marked taking all three spectra recorded, into consideration. In all, eight lines due to rovibronic transitions were found in this region [6-10]. Data for all the recorded spectra is available with the authors.

\section{Conclusion}

FT-Raman scattering result using an Nd: YAG laser excitation for the fullerene $\left(\mathrm{C}_{60}\right)$ molecule were studied and new vibrational transitions and formation of new compounds established from the recorded spectrum. Several lines are newly reported for the first time here. The new intense, or the not-so-intense lines are attributed to Stokes and anti-Stokes shifts respectively.

\section{Acknowledgement}

The authors are thankful to the management of Sri Sairam Engineering College, the Principal and the Dean (R \& D), for encouraging us to participate in International Conferences abroad. The authors are also thankful to the DST, Government of India, and SAIF, IIT, Madras for providing instrumentation facilities to record our spectrum.

\section{References}

1. Kroto HW, Heath JR, O'Brien SC, Curl RF, Smalley RE (1985) C 60 buckminsterfullerene. Nature 318: 162-163.

2. Raman CV (1928) A new radiation. Indian J. Phys. 2: 387-398. 
Citation: Subramaniam TY, Premanand P (2018) Infrared Laser Excitation to Study Transitions in Fullerene (C60) Molecule. J Laser Opt Photonics 5: 189. doi: 10.4172/2469-410X.1000189

Page 5 of 5

3. Oswaw E (2011) Perspectives of Fullerene nanotechnology. Springer, Netherlands, pp: 275-280.

4. Dresselhaus MS, Dresselhaus G, Eklund PC (1996) Science of Fullerenes and Carbon Nano tubes. Academic Press, San Diego.

5. Herzberg G (1939) Molecular spectra and molecular structure. II. Infrared and Raman spectra of polyatomic molecules Van Nostrand.

6. Kuzmany H, Pfeiffer R, Hulman M, Kramberger C (1824) Raman spectroscopy of fullerenes and fullerene-nanotube composites. Philos Trans A Math Phys Eng Sci Nov 15: 362
7. Dresselhaus MS, Dresselhaus G, Eklund PC (1996) Raman Scattering in Fullerenes. J Raman Spectrosc 27: 351-371.

8. Kataura H, Maniwa Y, Kodama T, Kikuchi K, Hirahara K et al. (2002) Fullerene-peapods: synthesis, structure, and Raman spectroscopy. AIP Conference Proceedings 591: 251.

9. Dresselhaus MS, Jorio A, Saito R (2010) Characterizing Graphene, Graphite, and Carbon Nanotubes by Raman Spectroscopy. Annu Rev Condens Matter Phys 1: 89-108.

10. Maidment L, Schunemann PG, Reid DT (2016) Molecular fingerprint-region spectroscopy from 5 to $12 \mu \mathrm{m}$ using an orientation-patterned gallium phosphide optical parametric oscillator. 41: 4261-4264. 